\title{
PROGRESS IN DEVELOPMENT OF AN INTERFEROMETRIC OIL MANOMETER
}

\author{
S. Ehlers ${ }^{1,2}$, W. Sabuga ${ }^{1,3}$ \\ ${ }^{1}$ Physikalisch-Technische Bundesanstalt, Bundesallee 100, Germany, ${ }^{2}$ sven.ehlers@ptb.de,${ }^{3}$ wladimir.sabuga@ptb.de
}

\begin{abstract}
:
At PTB, a new interferometric oil manometer for the measurement of pressures below $2 \mathrm{kPa}$, is in development. This paper describes how the main quantities, length and liquid density, are determined, both $i n$-situ, the latter for the first time in a primary pressure standard. The key features of both methods are presented.
\end{abstract}

Keywords: primary pressure standard; low pressure; liquid column manometer; interferometric length measurement; liquid density measurement

\section{INTRODUCTION}

Low pressures, between $1 \mathrm{~Pa}$ and a few $\mathrm{kPa}$, are measured in high-tech industries, such as pharmaceutical productions, clean room applications e.g. in semi-conductor and solar cell production, and the aviation and the space industry.

To guarantee reliability, efficiency and quality of those processes, pressure standards are used. National Metrology Institutes as integral part of the worldwide quality system use standards, ideally, that are primary ones, then offering smallest uncertainties, because of being directly traceable to the International System of Units (SI).

In dependence on the pressure mode, typical instruments in the described pressure range are, expansion systems, pressure balances with conical or spherical non-rotating piston, force-balanced piston gauges, diving-bell or liquid column manometers. Also, new developments based on optical methods, e.g. pressure measurement by determining the refractive index and, from this, density of the pressure transmitting medium at a certain temperature level should be remarked [1], even though, at this time, no examples of practical application of such standards as reference in calibrations are known.

Among all those instruments, pressure balances with non-rotating piston, such as force-balanced piston gauges, when using techniques recently made available [2], could be characterized as primary standards, by determining the dimensions of the piston-cylinder geometry in combination with the description of the flow conditions inside the pistoncylinder gap. However, in the most customeroriented calibrations of such force-balanced piston gauges, they are calibrated as secondary standards against another pressure standard, such as a deadweight pressure balance at pressures above few kilopascals, or liquid-column manometers in the lower pressure range.

As discussed below, liquid-column manometers (LCMs) present a preferable alternative to pressure balances for a primary realisation of the pressure unit in the pressure range below few kilopascals. The LCMs use fundamental, well-known physics, and, through the measurement of input quantities: length, density and gravity acceleration, they are directly traceable to the SI.

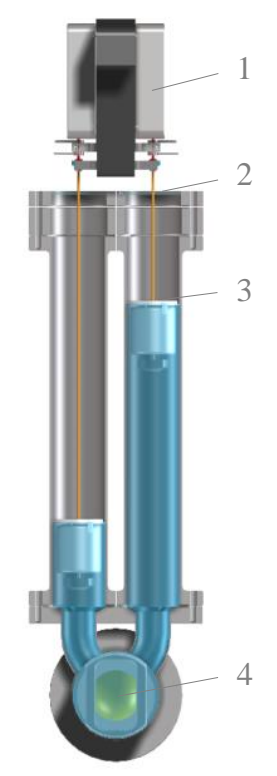

Figure 1: U-tube main assembly consisting of the differential interferometers (1), viewports (2), pool floats (3) and the density standard (4).

The liquid column manometer in development at PTB, its u-tube assembly is presented in Figure 1, is characterized by the first time integration of an in-situ density measurement, which enables the use of new liquids, like vacuum oils, offering, in comparison to mercury, another common liquid used for LCMs, an up to factor 16 lower density, and, therefore, 
increasing the pressure sensitivity significantly. In combination with an interferometer that uses the direct reflection of the laser light on the liquids free surface for the length measurement, the height difference of the two columns can be measured with a high accuracy.

\section{LIQUID COLUMN MANOMETRY}

Making use of the physical connection between the measured height difference of two liquid columns in a u-shaped tube and the applied pressure difference, has a long history in pressure metrology going back till Torricelli's invention of the barometer in 1643.

Several National Metrology Institutes have developed liquid column manometers. Typically, those instruments cover the barometric pressure range, but especially in the last decades, they were also characterized as owning a measurement range starting from around $1 \mathrm{~Pa}$ and ending at a few kilopascals or lower [3-6].

The pressure difference $\Delta p$ and the height difference of the liquid columns $\Delta h$ stand in connection with the gravitational acceleration $g$ and the liquid density $\rho_{l}$. As for $g$, it is easily possible to determine it once, onsite, using transportable gravimeter standards, on a relative uncertainty level of $10^{-7}$. So that the most important factor for the measurement characteristics of the instrument is the choice of a manometric liquid. Indeed, from (1) it can be seen that the LCM sensitivity to pressure changes is higher, the lower the liquid density is.

$\Delta p=\rho_{l} g \Delta h$.

Among manometric liquids, mercury is quite common [7-10] because of its elemental purity and stable density, but the accuracy of the pressure measurement suffers from the high mercury vapour pressure of around $0.2 \mathrm{~Pa}$, and applicability of mercury manometers is nowadays restricted primarily due to mercury's poisonousness.

Therefore, in the PTB approach, vacuum oils were considered as the best candidates as a manometric liquid inside the new interferometric oil manometer. Based on the results of the study of their properties [11], an Alpha-olefin, namely Edwards 45, usually used for vacuum pumps, was chosen. When being used in the manometer, a thermal control by a thermostatic bath is needed due to the oil's thermal expansion coefficient of around $0.7 \mathrm{ppm} / \mathrm{mK}$ (1 ppm $\left.\triangleq 10^{-6}\right)$. In addition, the liquid has a very low vapour pressure of $10^{-5} \mathrm{~Pa}$ and a density of around $830 \mathrm{~kg} / \mathrm{m}^{3}$, 16 times lower than that of mercury, which together offer a much better measurement capability of low pressures compared with that of mercury manometers.

The instrument uses two homodyne laser interferometers to detect the displacement of the liquid columns. The light of the Ne-He laser of the interferometer system is directly reflected from the liquid surface, built at the liquid-gas interface in each tube. This has advantage of an accurate detecting the oil surface's height position, without any disturbance which takes place in some alternative systems with a mirror carrier on the oil surface. However, the direct interferometric detecting of the oil surface is challenging because of a low oil reflectivity of about $4 \%$. Moreover, to prevent liquid surface waves, originating from the tube walls, reaching the surface's centre, where the laser beam is reflected, a so-called pool-float is used in each column.

\section{LENGTH MEASUREMENT}

To measure the displacement of the liquid's surfaces in each arm of the liquid column manometer, two individual differential interferometers, of type SP 2000 DI produced by SIOS Meßtechnik Gmbh, Germany are used. The differential principle - each laser interferometer sends two beams, one to the oil surface and the other to the tube window - allows for minimising the influence of ambient air's refractive index changes Figure 2. Furthermore, with the use of two interferometers, it is possible to measure the displacement of the oil surface in each column individually and independently of the liquid exchange between both legs of the LCM. The measurement always starts from the zero height difference, when both pressures in the two arms of the manometer are equalised. In the interferometers used, all parts involved in the displacement measurement are thermally passive elements, which is achieved by means of a fibre coupling of the He-Ne laser system.

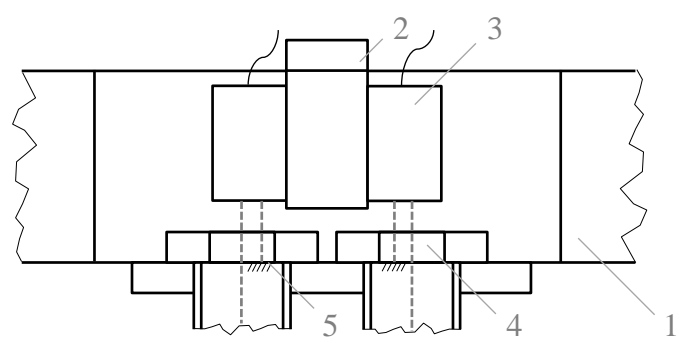

Figure 2: Interferometer setup containing the granite structure (1), stainless steel interferometer carrier (2), differential interferometers (3), viewports (4), reflective coating (5).

The interferometer system delivers a $0.1 \mathrm{~nm}$ resolution in length measurement, which, in an ideal case, would result in a $1 \mu \mathrm{Pa}$ pressure resolution. Additional influences impact the real pressure resolution, e.g. mechanical vibrations, so, in the practical operation of the instrument, the resolution will be lower. Experiments were carried out to test the resolution and the stability of the interferometric measurement of the oil surface's height. In the experiments, a test setup was used, arranged on top of the heavy granite structure of the LCM, and thermally shielded by foam plates, and the vacuum 
oil's surface was used as a mirror for the measurement beam of the interferometer. In Figure 3, the measurement signal of the laser interferometer recorded over the time of $20 \mathrm{~s}$ is shown. There, a scatter in the signal of about $2 \mathrm{~nm}$ and signal's temporal fluctuations with an amplitude of up to $5 \mathrm{~nm}$ can be identified - the low-frequent fluctuations are most likely related to the light structure of the aluminium test setup. Further tests, with the interferometers in their final position, coupled to the granite main structure of the LCM, will be performed. Nevertheless, by conservatively comparing the minimum and maximum values of the interferometer signal during the time of $20 \mathrm{~s}$, the difference between two signals is never bigger than $10 \mathrm{~nm}$, resulting in a resolution of lower than $0.1 \mathrm{mPa}$ in the unit of pressure.

Considering that the measurement beam, along the tube, is passing also the pressure transmitting media, which is usually nitrogen, a correction for the influence of the refractive index [6] needs to be applied. This correction can be calculated very accurately, as the tube assembly is temperature controlled by a thermostatic bath and the pressure inside the manometer can be easily estimated with an accuracy of a few pascals.

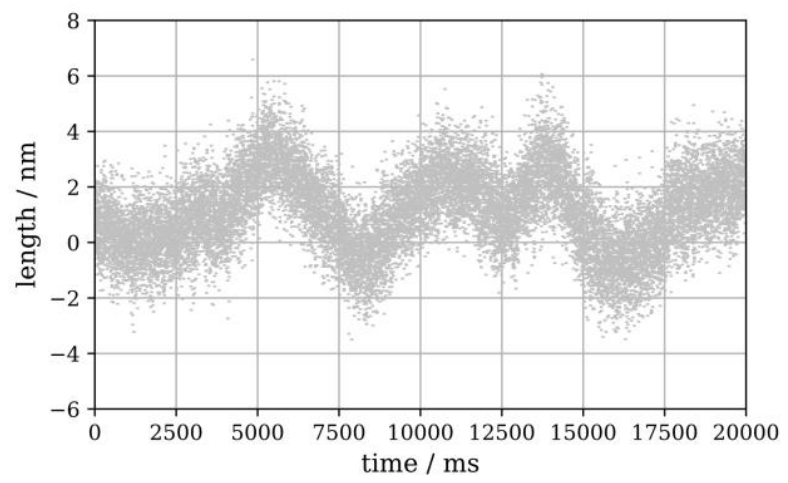

Figure 3: Length signal of a differential interferometer, when the measurement beam is reflected from the vacuum oil's surface, recorded with a sampling rate of $1 \mathrm{kHz}$.

As the measurement beam of the interferometer is reflected from the vacuum oil's liquid surface, a potential signal loss caused by waves that scatter the beam, especially when the height of the column changes, needs to be prevented. Therefore, a special pool float made of polytetrafluoroethylene (PTFE) is used in each tube leg to stabilise the oil surface. The pool float, whose sketch is presented in Figure 4, is supported by a hollow stainless-steel bouncy body, which defines such a position of the pool float at which its pool bottom lies by few millimetres beneath the liquid free surface. To enable the formation of a free liquid surface inside the pool at the height corresponding to column's hydrostatic pressure, the inner pool volume is always in connection to the surrounding liquid, even though, surface waves being built at the liquid - tube wall boundary are prevented to reach the inner pool. To get the oil surface shape within the pool as flat as possible, the inner walls of the pool are machined with the angle corresponding to the oil-PTFE wettability angle. Herewith, the oil surface is nearly horizontal at the wall contact perimeter, and the flatness of the oil layer in the pool is guaranteed.

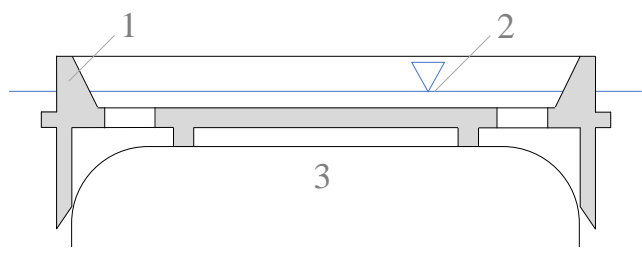

Figure 4: Pool float setup consisting of the PTFE pool float (1), free surface of the vacuum oil (2) and buoyancy body (3).

\section{LIQUID DENSITY DETERMINATION}

By the use of vacuum oils inside the liquid column manometers, on one hand, one receives an excellent pressure resolution and optimal conditions for absolute pressure measurements, as the liquid's vapour pressure is negligibly small. On the other hand, the system needs stable spatial and temporal thermal conditions, and, in addition, vacuum oils easily absorb gases, which influence the liquid density.

In order to achieve a relative uncertainty of a few ppm for the liquid density, in spite of the effect of the gas absorption and other instability effects, the idea came up to measure the density, for the first time in a liquid column manometer, in-situ, using the temperature-of-flotation method [11].

For determining the liquid density with this method, a solid density standard, whose density is precisely known and similar to that of the liquid, is immersed in the liquid. Then, the temperature of the whole setup, with the help of a thermostatic bath, is changed in a way, that the solid density standard comes in hydrostatic equilibrium with the surrounding liquid freely floating in a certain position in it.

Under this condition, and especially this temperature, the density of the standard and those of the liquid are the same, and, therefore, known.

The practical realisation of this method is possible because, in the case of the chosen vacuum oil, the volumetric thermal expansion coefficient of the oil [11] is by about factor 29 higher than that of the solid density standard, so that the oil density reacts much more sensitively to temperature changes.

For the application in the liquid column manometer, a hollow titanium sphere was manufactured as the density standard, which is nominally $100 \mathrm{~cm}^{3}$ in volume. Titanium was chosen as a sphere material because of its low density and low thermal expansion coefficient. 
For the density standard, two thin-walled halfspherical shells where manufactured, which then were laser welded. After that, a final production step was undertaken in PTB's department "Scientific Instrumentation", which has instrumentation and great experience in polishing spherical objects, so that the density of the sphere - solid density standard - was adopted to the density of the liquid. Figure 5 presents the titanium density standard after its final processing.

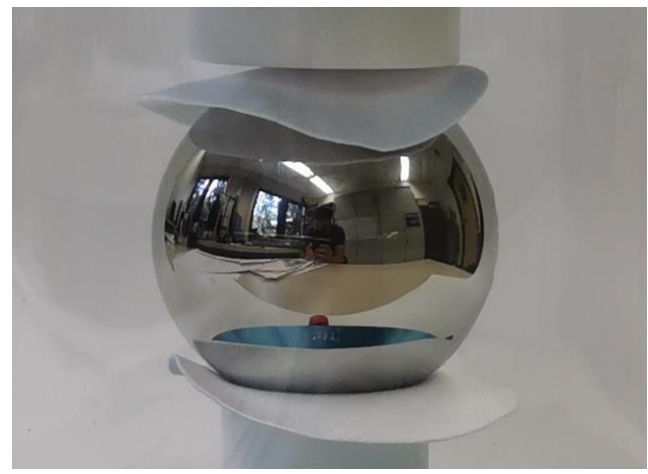

Figure 5: PTB's titanium density standard DS1, owning a nominal volume of $100 \mathrm{~cm}^{3}$.

At PTB, in the department "Mass", the density of solids, like the titanium density standard built, could be determined by hydrostatic weighing in comparison to silicon density standards, with a relative standard uncertainty at $20{ }^{\circ} \mathrm{C}$ of below $4 \times 10^{-6}$. With this measurement technique, the density of the titanium sphere will be determined with a comparable uncertainty, which fully satisfies the requirement for the oil density measurement in the interferometric oil manometer.

\section{SUMMARY}

In the development of the new interferometric liquid column manometer of PTB, designed for the measurement of low absolute and gauge pressures below $2 \mathrm{kPa}$, progress was made. The instrument capability in measuring the main relevant quantities - length of the liquid columns and the density of liquid, both directly traceable to the SI - was evaluated. The unique feature of the new manometer is its capability to measure the density of the liquid in-situ. With special emphasis on the key issues, such as using the direct reflection of the laser light from the free surface of the oil under low reflectivity conditions, stabilisation of the oil free surface with the help of a pool float, and also the first-time integration of a liquid density measurement, pressure measurement with a standard uncertainty below $1 \mathrm{mPa}+18 \mathrm{ppm}$ appears feasible.

\section{REFERENCES}

[1] K. Jousten, J. Hendricks, D. Barker, K. Douglas, S. Eckel, P. Egan, J. Fedchak, J. Flügge, C. Gaiser, D. Olson, J. Ricker, T. Rubin, W. Sabuga, J. Scherschligt, R. Schödel, U. Sterr, J. Stone, and G. Strouse, "Perspectives for a new realization of the pascal by optical methods", Metrologia, vol. 54, no. 6, pp. 146-161, Nov. 2017.

[2] A. S. Hashad, S. Ehlers, O. Jusko, and W. Sabuga, "Characterization of a force-balanced piston gauge as a primary pressure standard", Measurement, vol. 131, pp. 723-729, 2019.

[3] M. Ueki and A. Ooiwa, "A Heterodyne Laser Interferometric Oil Manometer," Metrologia, vol. 30, no. 6, pp. 579-583, Jan. 1994.

[4] K. F. Poulter and P. J. Nash, "An interferometric oil micromanometer," Journal of Physics E: Scientific Instruments, vol. 12, no. 10, pp. 931-936, Oct. 1979.

[5] Y. Li, Y. Yang, J. Wang, and J. Sun, “A new primary standard oil manometer for absolute pressure up to 10 kPa", Metrologia, vol. 52, no. 1, pp. 111-120, Jan. 2015.

[6] I. V. Sadkovskaya and A. I. Eichwald, "A laser interferometric oil manometer as the primary standard for absolute pressure in the range $1-1000$ Pa", Journal of Physics: Conference Series, vol. 100, no. 9, p. 092006, Mar. 2008.

[7] I. V. Sadkovskaya and A. I. Eykhval'd, "Laser Interferometric Mercury Manometer of State Primary Standard Get 101-2011", Measurement Techniques, vol. 57, no. 11, pp. 1238-1244, Feb. 2015.

[8] J. Jäger, "Use of a Precision Mercury Manometer with Capacitance Sensing of the Menisci," Metrologia, vol. 30, no. 6, pp. 553-558, Jan. 1994.

[9] A. P. Miiller, C. R. Tilford, J. H. Hendricks, “A low differential-pressure primary standard for the range 1 Pa to $13 \mathrm{kPa}$ ", Metrologia, vol. 42, no. 6, pp. S187S192, Nov. 2005.

[10] P. L. M. Heydemann, C. R. Tilford, R. W. Hyland, "Ultrasonic manometers for low and medium vacua under development at the National Bureau of Standards", Journal of Vacuum Science and Technology, vol. 14, no. 1, pp. 597-605, Jan. 1977.

[11] S. Ehlers, J. Könemann, O. Ott, H. Wolf, J. Šetina, A. Furtado, and W. Sabuga, "Selection and characterization of liquids for a low pressure interferometric liquid column manometer", Measurement, vol. 132, pp. 191-198, 2019. 